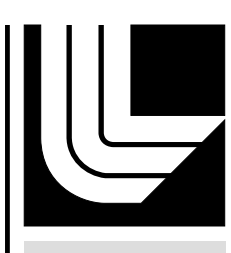

LAWRENCE LIVERMORE NATIONAL LABORATORY

Prospects for achieving high dynamic compression with low energy

M. R. Armstrong, S. Bastea, J. Crowhurst, W. Howard, J. Zaug, A. Goncharov

August 17, 2012

Applied Physics Letters 
This document was prepared as an account of work sponsored by an agency of the United States government. Neither the United States government nor Lawrence Livermore National Security, LLC, nor any of their employees makes any warranty, expressed or implied, or assumes any legal liability or responsibility for the accuracy, completeness, or usefulness of any information, apparatus, product, or process disclosed, or represents that its use would not infringe privately owned rights. Reference herein to any specific commercial product, process, or service by trade name, trademark, manufacturer, or otherwise does not necessarily constitute or imply its endorsement, recommendation, or favoring by the United States government or Lawrence Livermore National Security, LLC. The views and opinions of authors expressed herein do not necessarily state or reflect those of the United States government or Lawrence Livermore National Security, LLC, and shall not be used for advertising or product endorsement purposes. 


\title{
Prospects for achieving high dynamic compression with low energy
}

Michael R. Armstrong*, Jonathan C. Crowhurst, Sorin Bastea, William M. Howard, and Joseph M. Zaug

Physical and Life Sciences Directorate, Lawrence Livermore National Laboratory, Livermore, California 94550, USA

\author{
Alexander F. Goncharov \\ Geophysical Laboratory, Carnegie Institute of Washington, Washington, DC 20015, USA \\ *email: armstrong30@llnl.gov
}

\begin{abstract}
Laser driven dynamic compression experiments, may, in materials with picosecond equilibration times, be possible with orders of magnitude less drive energy than currently used. As we show, the compression energy for geometrically similar experiments varies as the third power of the time scale of compression. For materials which equilibrate and can be characterized on picosecond time scales, the compression energy can be orders of magnitude smaller than the 1-100 nanosecond scale time scale of many current experiments. Recent experiments have demonstrated that dynamically compressed materials can equilibrate and be characterized on hundreds of picosecond time scales, consistent with this proposal.
\end{abstract}


Substantial experimental resources have been devoted to the dynamic compression of materials to extremely high (multiple Mbar) pressure over short (typically sub-ms) time scales $^{1-6}$. Dynamic compression experiments use material impact- or laser driven compression waves, where the speed (or speed distribution) of the wave front and the corresponding speed of material behind the wave front may be used (via conservation of mass and momentum) to infer the density and pressure of the dynamically compressed state. In practice, such experiments often employ shock waves. Ideally, a shock wave is a large amplitude, steady monotonic compression wave which propagates faster than the speed of sound in the uncompressed material and achieves an equilibrium final state over a material dependent rise time which, in the absence of kinetically limited material transformations, can have $\sim$ picosecond duration ${ }^{2}$.

For the determination of an equilibrium property, in principle the experiment does not need to be much longer than the time required to reach equilibrium and characterize the equilibrium state (via wave and particle speed measurements). Historically, shock wave experiments use compression times much longer than a nanosecond, which is a typical time resolution. Within the last two decades, methods to characterize shock waves with picosecond time resolution have been developed ${ }^{7-11}$, resulting in the measurement of enormous elastic compression on short time scales ${ }^{12-14}$, and the measurement of a tens of picosecond scale plastic shock rise time in aluminum ${ }^{12}$. These experiments require orders of magnitude less compression energy than longer time scale experiments to obtain and characterize comparable thermodynamic conditions, because the volume of compressed 
material is much smaller (i.e. pL, compared to typically more than $0.1 \mu \mathrm{L}$ for larger scale work). Here we outline principles which establish a lower bound to the reduction of length, time, and energy scales in dynamic compression experiments, and show that some experiments might be performed with orders of magnitude less energy than used in longer time scale experiments.

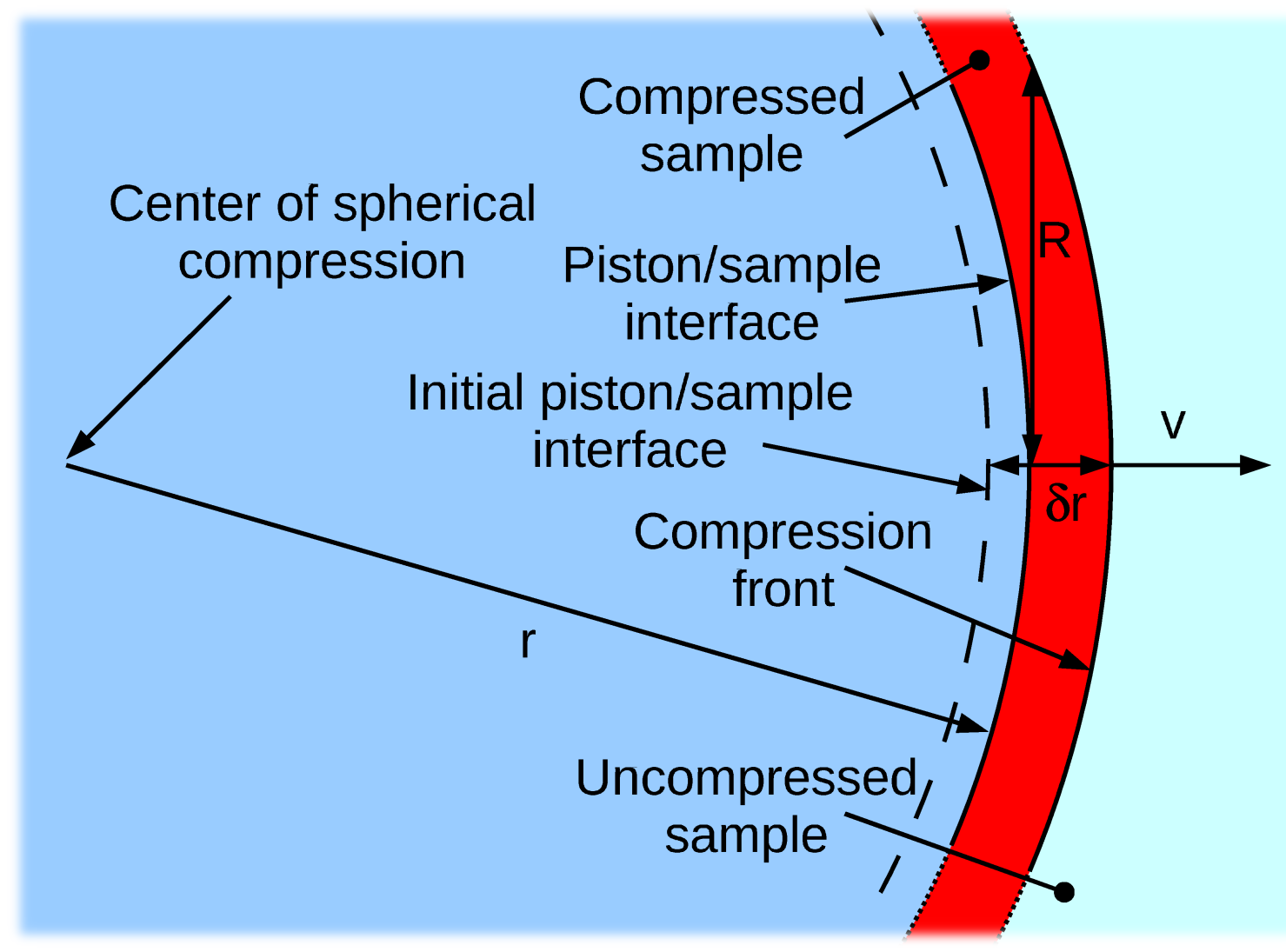

Figure 1: A cross-sectional schematic of a spherical dynamic compression experiment from the side, with radius of curvature of the compression front, $r$, transverse radius, $R$, and the propagation distance of the compression front over the duration of the experiment, $\delta r$. The compression front and piston/sample interface propagate to the right, and the compression front has speed $\mathrm{v}$. We assume the piston instantaneously jumps to a radial, constant particle velocity at $t=0$, so that $\delta \mathrm{r}=\mathrm{v} \mathbf{t}_{\mathrm{c}}$.

In a dynamic compression experiment, the laser drive energy is roughly proportional to the compression energy, which is proportional to the volume of compressed material, 
$E_{c} \propto V_{c}$. The volume of material to be compressed (see Fig. 1) is given by:

$$
V_{c} \approx v t_{c} C R^{2}=\delta r C R^{2}
$$

where $t_{c}$ is the compression time, $v$ is the average speed of the compression wave, $\mathrm{R}$ is the radius of the compressed region transverse to the direction of travel of the compression wave, and $\mathrm{C}$ is a constant which accounts for the definition of R. For spherical compression, eq. 1 is accurate only when $\delta r \ll r$, and here $\delta r=v t_{c}$. R may be defined as, for instance, the half width of the transverse particle speed profile of the compression front, but the results below do not vary substantially (compared to an order of magnitude) over reasonable definitions of R.

In principle, neither obtaining extreme conditions nor characterizing the final state requires $1 \mathrm{D}$ compression. Nonetheless, we consider the deviation of finite aspect ratio (i.e. finite spot size) experiments from 1D compression to establish a common reference for the comparison of experiments at different scales. We define the aspect ratio, A, to be proportional to $R / \delta r$ (see Fig. 1), where the constant of proportionality depends on the definition of R. Henceforth we assume a definition for R has been chosen to compare experiments. We assume a spherical compression front, with the understanding that, 1) 1D planar compression is the limit of spherical compression as the aspect ratio approaches infinity, and 2) spherical compression fronts approximate those generated by a finite spot size in laser driven experiments. The deviation of the compression wave speed obtained in a quasi-1D experiment (i.e. finite laser drive spot size) from that obtained in ideal 1D compression has an upper bound given by ${ }^{15}$ : 


$$
\left|\frac{\delta v}{v}\right|<\left|\frac{\delta r}{r}\right| \propto \frac{1}{A^{2}},
$$

where $\delta v$ is the deviation in compression wave speed from the ideal 1D case, $\mathrm{r}$ is the radius of curvature of the compression wave front (see Fig. 1), the proportionality is approximately valid for large A, and $\delta r / r$ is a function of the aspect ratio only (for a given definition of $\mathrm{R}$ ). So, the deviation of the compression wave speed (and related deviations in thermodynamic properties) from the $1 \mathrm{D}$ value is constant for a given aspect ratio. Effectively, experiments with the same aspect ratio (or planarity) are equivalent, assuming the compression time is sufficient to obtain and characterize the relevant equilibrium compressed states.

The aspect ratio is a measure of the planarity of the wave over the duration of the experiment, and is related to another metric for the planarity ${ }^{16,17}$,

$$
\theta_{\text {bow }} \propto \frac{1}{A}
$$

Generally, $\theta_{\text {bow }}$ is used to correct time of flight measurements for non-planar wave propagation in longer time scale experiments, whereas here we use the aspect ratio to estimate the deviation of measured wave speeds from ideal 1D compression.

Rewriting eq. 1 in terms of the aspect ratio,

$$
E_{c} \propto V_{c} \approx t_{c}^{3} v^{3} C^{\prime} A^{2}
$$

(where $\mathrm{C}^{\prime}$ now also accounts for the constant of proportionality in the definition of $\mathrm{R}$ ), allows direct comparison of compression energy as a function of compression time for 
geometrically similar experiments. For a constant aspect ratio, sample, and final thermodynamic state, the compression energy scales with the third power of the compression time, $t_{c}$.

The compression time must be at least the time required for the sample to reach the final, equilibrated thermodynamic state plus the time required to characterize that state, and we may define a measurement efficiency for compression experiments as the ratio of the minimum compression time divided by the actual compression time. In a shock wave experiment, the equilibration time can be very short, in the picosecond range ${ }^{2}$. Recent observations of shock waves in aluminum ${ }^{12}$ at shock pressures above 40 GPa measure the steady state plastic rise time to be less than 30 picoseconds. Resolution limited measurements of the time for shocks to merge in multiple shock experiments in initially cryogenic deuterium indicate a single shock rise time of less than 22 picoseconds ${ }^{6}$. Further, measurements of wave speeds have been demonstrated in many cases with sub10 ps time resolution ${ }^{7-10,12}$, and, in conventional experiments, with sub-100 ps time resolution ${ }^{3,6}$.

To illustrate the potential advantage of a reduction in scale, consider Hicks et al. ${ }^{3}$, where a 3.7 nanosecond, < $3 \mathrm{~kJ}$ energy pulse was used to shock compress liquid cryogenic deuterium. Assuming a total compression time of about 185 ps (20x shorter than 3.7 ns, but sufficient for equilibration and wave speed measurement), at a constant aspect ratio (i.e. the spatial scale of the experiment must also be reduced by a factor of 20), the 
compression pulse energy may be reduced to less than $375 \mathrm{~mJ}$, almost $10^{4}$ less laser drive energy.

We also note the potential to substantially reduce the scale of quasi-isentropic, ramp compression experiments. In particular, shock waves in weakly interacting materials (such as van der Waals liquids) can have sub-ps rise times (from simulations ${ }^{18}$ ), implying picosecond equilibration times. Ramp compression experiments typically apply compression over much more than 1 ns, generally between 10-100 ns, to avoid the formation of shock waves during the experiment, yet for picoseond equilibration times, quasi-isentropic compression might be achieved even for sub-ns compression times. Consistent with the above example, a 10x reduction in compression time corresponds to a factor of 1000 in laser drive energy. 


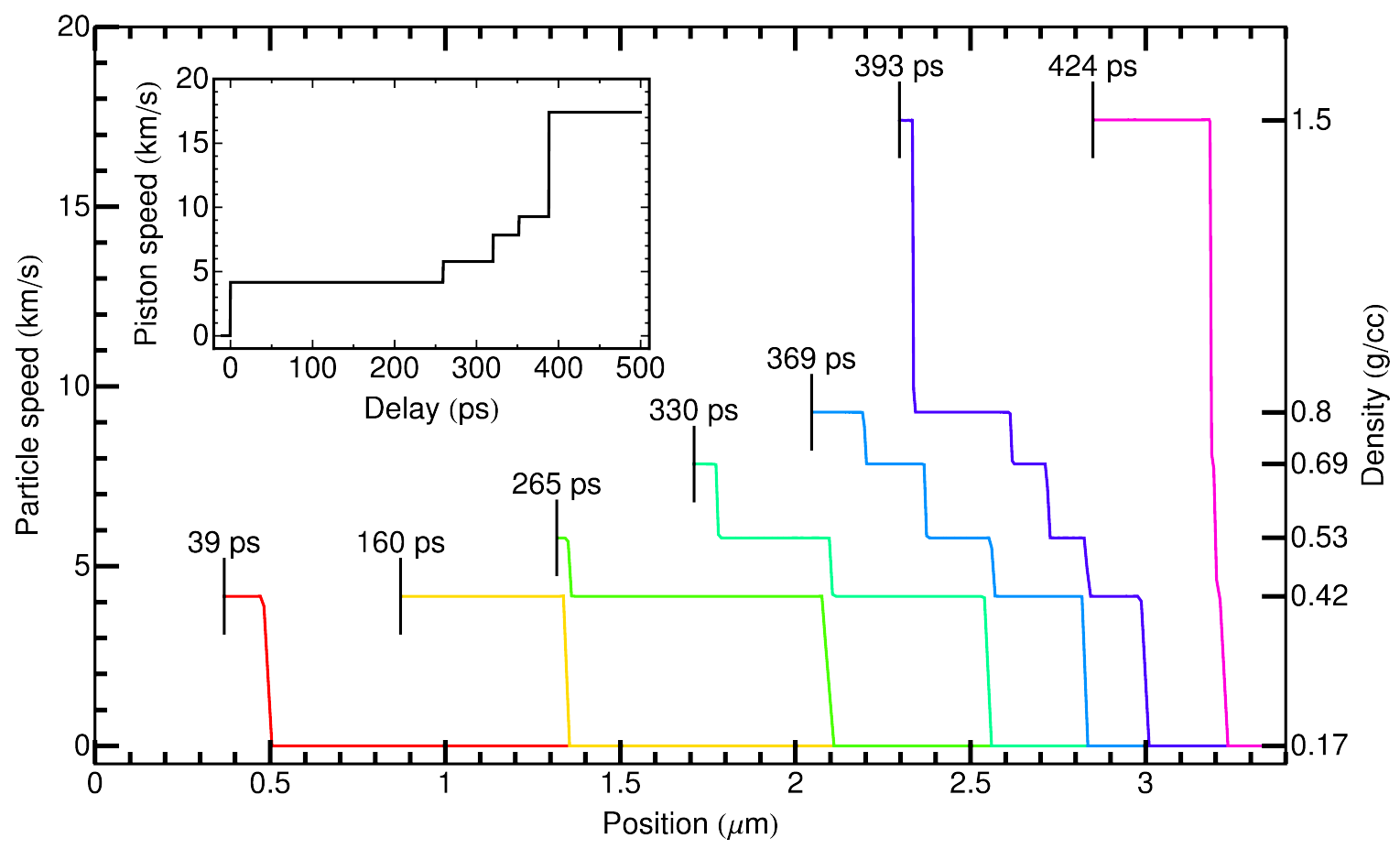

Figure 2: The results of a 2D hydrodynamics simulation of the wave evolution for multiple shock wave compression of cryogenic liquid deuterium from $20 \mathrm{~K}$ initial temperature with a radially symmetric Gaussian particle speed distribution with a full width half maximum of $90 \mu \mathrm{m}$. Particle speed distributions for different times (as labeled) along the axis of symmetry are shown. Vertical black lines in the main plot show the piston position and are labeled by the time for each plot. Density is labeled for each step on the right side axis. The shock fronts are designed to converge at a single depth. The piston speed as a function of time along the axis of symmetry is shown in the inset. The final temperature from the simulations is $\sim 3600 \mathrm{~K}$, whereas the temperature of shock loaded deuterium at the same final pressure ( $135 \mathrm{GPa}$, also from simulations) is $>30,000 \mathrm{~K}$.

A 2D hydrodynamics simulation of multiple shock compression of cryogenic liquid deuterium (shown in Fig. 2) illustrates the basic feasibility of sub-ns quasi-isentropic compression. The multi-physics code, ALE3D ${ }^{19}$, which can comprise several classes of phenomena, including hydrodynamics, thermal transport, equations of state, and reaction chemistry, was used to perform the simulations. The mathematical formulation is based on an Operator-Split method and involves an arbitrary Lagrangian-Eulerian (ALE) approach in a 3-D/2-D configuration. In these simulations we have not included the 
effects of reaction chemistry. For these simulations we used a Quotidian ${ }^{20}$ equation of state for the deuterium. This equation of state is used in the form of a table and for deuterium it is valid in the regime of temperature from $1 \mathrm{~K}$ to $2 * 10^{9} \mathrm{~K}$ and in the range of density from $10^{-10}$ to $10^{4} \mathrm{~g} / \mathrm{cm}^{3}$.

From the simulation, the total compression is $\sim 9 \mathrm{x}$ over about $3 \mu \mathrm{m}$ of initially uncompressed sample thickness, along the axis of symmetry. The shock wave speeds along the axis of symmetry are well within $1 \%$ of shock wave speeds for ideal $1 \mathrm{D}$ compression, verifying that wave velocities in quasi-1D compression (at this aspect ratio) compare very well to wave velocities in ideal 1D compression, in principle. Wave velocities have been measured over $\sim 1 \mu \mathrm{m}$ spot sizes $^{10,12}$, so variation of the wave speed over the compression spatial profile is negligible for a $90 \mu \mathrm{m}$ full width half maximum spot size.

Using well-known scaling laws relating the ablator piston speed to the pump intensity ${ }^{21,22}$ and assuming a Gaussian spatial distribution of particle speeds with $90 \mu \mathrm{m}$ full width half maximum (sufficient to maintain an aspect ratio comparable to longer time scale work), this simulation indicates that $\sim 9 \mathrm{x}$ initial density can be obtained over $\sim 500$ ps with $\sim 200$ $\mathrm{mJ}$ of pump energy, which is substantially smaller than longer time scale experiments which achieve comparable final thermodynamic states ${ }^{3}$.

In summary, known picosecond equilibration times in materials coupled with picosecond 
time resolution available to modern diagnostics open a path to extreme conditions with orders of magnitude less drive energy than currently used. This may be achieved via compression on a time scale as much as $10-100 x$ closer to the minimum time required to obtain and characterize the equilibrium state.

We acknowledge useful conversations with L. E. Fried, W. J. Nellis, J. Eggert, R. Smith, B. Militzer, J. Carter, and J. Forbes. This work was performed under the auspices of the U.S. Department of Energy by Lawrence Livermore National Laboratory under Contract No. DE-AC52-07NA27344 with Laboratory directed Research and Development funding (11ERD039), as well as being based on work supported as part of the EFree, an Energy Frontier Research Center funded by the U.S. Department of Energy, Office of Science, Office of Basic Energy Sciences under Grant No. DESC0001057. 


\section{References}

${ }^{1}$ J. Lindl, Phys. Plasmas 2, 3933-4024 (1995).

${ }^{2}$ W.J. Nellis, Rep. Prog. Phys. 69, 1479-1580 (2006).

${ }^{3}$ D.G. Hicks, T.R. Boehly, P.M. Celliers, J.H. Eggert, S.J. Moon, D.D. Meyerhofer, and G.W. Collins, Phys. Rev. B 79, 014112 (2009).

4 J.E. Bailey, M.D. Knudson, A.L. Carlson, G.S. Dunham, M.P. Desjarlais, D.L. Hanson, and J.R. Asay, Phys. Rev. B 78, 144107 (2008).

${ }^{5}$ R. Smith, J. Eggert, A. Jankowski, P. Celliers, M. Edwards, Y. Gupta, J. Asay, and G. Collins, Phys. Rev. Lett. 98, 065701 (2007).

${ }^{6}$ T.R. Boehly, V.N. Goncharov, W. Seka, S.X. Hu, J.A. Marozas, D.D. Meyerhofer, P.M. Celliers, D.G. Hicks, M.A. Barrios, D. Fratanduono, and G.W. Collins, Phys. Plasmas 18, 092706 (2011).

${ }^{7}$ R. Evans, A.D. Badger, F. Falliès, M. Mahdieh, T.A. Hall, P. Audebert, J.-P. Geindre, J.C. Gauthier, A. Mysyrowicz, G. Grillon, and A. Antonetti, Phys. Rev. Lett. 77, 3359 (1996).

${ }^{8}$ A. Benuzzi-Mounaix, M. Koenig, J.M. Boudenne, T.A. Hall, D. Batani, F. Scianitti, A. Masini, and D. Di Santo, Phys. Rev. E 60, R2488-R2491 (1999).

${ }^{9}$ C.A. Bolme, S.D. McGrane, D.S. Moore, and D.J. Funk, J. Appl. Phys. 102, 033513 (2007).

${ }^{10}$ M.R. Armstrong, J.C. Crowhurst, S. Bastea, and J.M. Zaug, J. Appl. Phys. 108, 023511 (2010).

${ }^{11}$ M.R. Armstrong, J.C. Crowhurst, S. Bastea, and J.M. Zaug, in Proceedings of the 14th 
International Detonation Symposium (Coeur d'Alene, Idaho, 2010).

12 J.C. Crowhurst, M.R. Armstrong, K.B. Knight, J.M. Zaug, and E.M. Behymer, Phys. Rev. Lett. 107, 144302 (2011).

${ }^{13}$ S.I. Ashitkov, M.B. Agranat, G.I. Kanel’, P.S. Komarov, and V.E. Fortov, JETP Lett. 92, 516-520 (2010).

${ }^{14}$ V.H. Whitley, S.D. McGrane, D.E. Eakins, C.A. Bolme, D.S. Moore, and J.F. Bingert, J. Appl. Phys. 109, 013505 (2011).

${ }^{15}$ G.B. Whitham, Journal of Fluid Mechanics 4, 337-360 (1958).

${ }^{16}$ D.L. Paisley, S.-N. Luo, S.R. Greenfield, and A.C. Koskelo, Review of Scientific Instruments 79, 023902-023902-8 (2008).

${ }^{17}$ A.C. Mitchell and W.J. Nellis, Review of Scientific Instruments 52, 347-359 (1981).

${ }^{18}$ B.L. Holian, W.G. Hoover, B. Moran, and G.K. Straub, Phys. Rev. A 22, 2798-2808 (1980).

${ }^{19}$ A.L. Nichols, Users Manual for ALE3D, LLNL Technical Report LLNL-SM-433954 (Lawrence Livermore National Laboratory, 2010).

${ }^{20}$ R. More, K. Warren, D. Young, and G. Zimmerman, Phys. Fluids 31, 3059-3078 (1988).

${ }^{21}$ R. Decoste, S.E. Bodner, B.H. Ripin, E.A. McLean, S.P. Obenschain, and C.M. Armstrong, Phys. Rev. Lett. 42, 1673-1677 (1979).

${ }^{22}$ D.C. Swift and R.G. Kraus, Phys. Rev. E 77, 066402 (2008). 